\title{
Quantum theory of redshift in de Sitter expanding universe
}

\author{
Ion I. Cotăescu ${ }^{\mathrm{a}}(1)$ \\ West University of Timişoara, V. Pârvan Ave. 4, 300223 Timişoara, Romania
}

Received: 24 February 2021 / Accepted: 3 June 2021 / Published online: 27 June 2021

(C) The Author(s) 2021

\begin{abstract}
The quantum theory of the Maxwell free field in Coulomb gauge on the de Sitter expanding universe is completed with the technical elements needed for building a coherent quantum theory of redshift. Paying special attention to the conserved observables and defining the projection operator selecting the detected momenta it is shown that the expectation values of the energies of the emitted and detected photons comply with the Lemaitre rule of Hubble's law. Moreover, the quantum corrections to the dispersions of the principal observables and new uncertainty relations are derived.
\end{abstract}

\section{Introduction}

An important source of empirical data in the observational astrophysics is the light emitted by different cosmic objects whose redshifts encapsulate information about the cosmic expansion and possible peculiar velocities of the observed objects [1]. For understanding these two contributions one combined so far the Lemaître rule [2,3] of Hubble's law [4], describing the cosmological effect [5], with the usual theory of the Doppler effect of special relativity. Recently we proposed an improvement of this approach replacing the special relativity with our de Sitter relativity [6,7]. We obtained thus a redshift formula having a new term combining the cosmological and kinetic contributions in a non-trivial manner [8]. Moreover, we related the black hole shadow and redshift for the Schwarzschild [9] and Reissner-Nordstrom [10] black holes moving freely in the de Sitter expanding universe.

The next step might be the quantum theory of redshift but this was never considered because of the real or presumed difficulties in constructing the quantum theory of light in curved backgrounds. In fact there is nothing much in it since we have already the classical and quantum theory of the free Maxwell field on the de Sitter expanding universe [11] including the

\footnotetext{
a e-mail: i.cotaescu@e-uvt.ro (corresponding author)
}

de Sitter QED in the first order of perturbations [12]. Therefore, we may build a quantum theory of redshift exploiting this framework and solving the specific difficulties of this problem. We devote this paper to this goal constructing step by step the redshift theory from the classical level up to a new quantum approach able to reveal the quantum corrections and the uncertainty relations related to this effect.

The cornerstone here is the conformal covariance of the Maxwell equations in Coulomb gauge allowing us to take over all the results of special relativity in the comoving local charts (called here frames) with conformal coordinates of the de Sitter expanding universe [11]. In these frames the quantization of the Maxwell field can be done in canonical manner as in special relativity. The difference is that there is a richer algebra of isometry generators giving rise to more conserved quantities of the classical theory that become conserved one-particle operators after quantization [11]. Of special interest is the energy operator, which does not commute with the components of the conserved momentum generating new uncertainty relations [13].

On the other hand, the conformal coordinates are different from the physical ones which are of the Painlevé type [14] being related to the conformal ones through coordinate transformations depending on time. However, in the quantum theory these transformations change the time evolution picture as we have shown in Refs. [15-17]. Therefore, for avoiding this difficulty, we restrict ourselves to the conformal coordinates setting the initial conditions at the time $t_{0}$ when the scale factor $a\left(t_{0}\right)=1$ and the physical and conformal space coordinates coincide. Under such circumstances the physical effects may be studied by using exclusively the conserved one-particle operators.

In addition, we pay attention to a pair of sensitive technical problems which are crucial in our approach. The first one is related to the momentum-dependent phase of the plane wave solutions of the Maxwell equations which determines the form of the energy operator. Here we set for the first time the phase which guarantees the correct flat limit of our the- 
ory. The second problem is related to the detector measuring the redshift which has to select only the radiation emitted by a remote source. For doing so we assume that the detector filters the momenta in a desired domain of the momentum space whose associated projection operator helps us to derive the expectation values and dispersions of the measured observables.

We obtain thus a complete quantum theory of the redshift observed in the radiation emitted by a remote source without peculiar velocity. We show that the expectations values of the energies of the emitted and detected photons comply with the Lemaitre rule of Hubble's law, while the dispersions get new quantum corrections involved in a set of new uncertainty relations. However, it is less probable to identify such corrections in the astrophysical observations since these are very small in our actual expanding universe. Nevertheless, the methods developed here are important as these can be adapted to any spatially flat Friedmann-Lemaître-Robertson-Walker (FLRW) spacetime including those studied in the cosmology of early universe.

We start in Sect. 2 with a brief review of the de Sitter geometry defining the conserved quantities and introducing the conformal and physical coordinates. In the next section we revisit the classical theory of redshift pointing out the role of the conserved quantities in deriving the Lemaître equation. The Sect. 4 is devoted to the classical theory of the Maxwell field showing how by fixing a convenient phase we ensure the correct flat limit of the principal conserved quantities which become one-particle operators after the quantization performed in the next section. In the last part of this section we show how the wave packets can be measured by choosing a suitable projection operator for selecting the momenta of the modes which contribute to the expectation values of the principal conserved observables. The next section is devoted to the quantum redshift for which we derive the new quantum corrections and uncertainty relations. Finally we present some concluding remarks.

As here we develop a quantum approach, we introduce a special notation denoting by $\omega_{\mathrm{H}}=\sqrt{\frac{\Lambda}{3}} c$ the de Sitter Hubble constant (frequency) since $H$ is reserved for the energy or Hamiltonian operator [13]. Moreover, the Hubble time $t_{\mathrm{H}}=$ $\frac{1}{\omega_{\mathrm{H}}}$ and the Hubble length $l_{\mathrm{H}}=\frac{c}{\omega_{\mathrm{H}}}$ will have the same form in the natural Planck units with $c=\hbar=G=1$ we use here.

\section{2 de Sitter expanding universe}

The manifold in which we would like to study the quantum theory of redshift is the expanding portion $M_{+}$of the de Sitter spacetime $M$ known as the de Sitter expanding universe. The manifold $M$ may be defined as the hyperboloid of radius $1 / \omega_{\mathrm{H}}$ in the five-dimensional flat space- time $\left(M^{5}, \eta^{5}\right)$ of coordinates $z^{A}$ (labeled by the indices $A, B, \ldots=0,1,2,3,4)$ having the flat metric $\eta^{5}=$ $\operatorname{diag}(1,-1,-1,-1,-1)$. The frames $\{x\}$ of coordinates $x^{\mu}$ (with natural indices $\alpha, \mu, v, \ldots=0,1,2,3)$ can be introduced on $M$ or $M_{+}$giving the set of functions $z^{A}(x)$ which solve the hyperboloid equation,

$\eta_{A B}^{5} z^{A}(x) z^{B}(x)=-\frac{1}{\omega_{\mathrm{H}}^{2}}$,

where $\omega_{\mathrm{H}}$ is the Hubble de Sitter constant (frequency) in our notation. In what follows we consider the comoving frames with two sets of local coordinates, the conformal ones and the physical de Sitter-Painlevé coordinates.

The conformal coordinates $\left\{x_{\mathrm{c}}\right\}=\left\{t_{\mathrm{c}}, \mathbf{x}_{\mathrm{c}}\right\}$ are formed by the conformal time $t_{c}$ and the conformal Cartesian spaces coordinates $x_{\mathrm{c}}^{i}(i, j, k, \ldots=1,2,3)$, known as comoving space coordinates [18], for which we use the vector notation, $\mathbf{x}_{\mathrm{c}}=\left(x_{\mathrm{c}}^{1}, x_{\mathrm{c}}^{2}, x_{\mathrm{c}}^{3}\right) \in \mathbb{R}^{3} \subset M^{5}$. These are defined by the functions

$z^{0}\left(x_{\mathrm{c}}\right)=-\frac{1}{2 \omega_{H}^{2} t_{\mathrm{c}}}\left[1-\omega_{H}^{2}\left(t_{\mathrm{c}}{ }^{2}-\mathbf{x}_{\mathrm{c}}^{2}\right)\right]$,

$z^{i}\left(x_{\mathrm{c}}\right)=-\frac{1}{\omega_{\mathrm{H}} t} x_{\mathrm{c}}^{i}$

$z^{4}\left(x_{\mathrm{c}}\right)=-\frac{1}{2 \omega_{\mathrm{H}}^{2} t_{\mathrm{c}}}\left[1+\omega_{\mathrm{H}}^{2}\left(t_{\mathrm{c}}{ }^{2}-\mathbf{x}_{\mathrm{c}}^{2}\right)\right]$,

covering the expanding portion $M_{+}$for $t_{\mathrm{c}} \in(-\infty, 0)$ and $\mathbf{x}_{\mathrm{c}} \in \mathbb{R}^{3}$ while the collapsing part $M_{-}$is covered by similar frames with $t_{\mathrm{c}}>0$. In both these cases we have the same conformal flat line element,

$$
\begin{aligned}
\mathrm{d} s^{2} & =\eta_{A B}^{5} \mathrm{~d} z^{A}\left(x_{\mathrm{c}}\right) \mathrm{d} z^{B}\left(x_{\mathrm{c}}\right) \\
& =g_{\mu \nu}\left(x_{\mathrm{c}}\right) \mathrm{d} x_{\mathrm{c}}^{\mu} \mathrm{d} x_{\mathrm{c}}^{\nu}=\frac{1}{\omega_{\mathrm{H}}^{2} t_{\mathrm{c}}{ }^{2}}\left(\mathrm{~d}{t_{\mathrm{c}}}^{2}-\mathrm{d} \mathbf{x}_{\mathrm{c}} \cdot \mathrm{d} \mathbf{x}_{\mathrm{c}}\right) .
\end{aligned}
$$

Here we restrict ourselves to the expanding portion $M_{+}$ which is a plausible model of our expanding universe.

The de Sitter-Painlevé coordinates $\{x\}=\{t, \mathbf{x}\}$ on the expanding portion $M_{+}$can be introduced directly by substituting

$t_{\mathrm{c}}=-\frac{1}{\omega_{\mathrm{H}}} e^{-\omega_{\mathrm{H}} t}, \quad \mathbf{x}_{\mathrm{c}}=\mathbf{x} e^{-\omega_{\mathrm{H}} t}$,

where $t \in(-\infty, \infty)$ is the proper or cosmic time while $x^{i}$ are the physical Cartesian space coordinates of an observer staying at rest in origin. Then the line element reads

$$
\begin{aligned}
\mathrm{d} s^{2} & =g_{\mu \nu}(x) \mathrm{d} x^{\mu} \mathrm{d} x^{\nu} \\
& =\left(1-\omega_{\mathrm{H}}^{2} \mathbf{x}^{2}\right) \mathrm{d} t^{2}+2 \omega_{\mathrm{H}} \mathbf{x} \cdot \mathrm{d} \mathbf{x} \mathrm{d} t-\mathrm{d} \mathbf{x} \cdot \mathrm{d} \mathbf{x} .
\end{aligned}
$$

Notice that this frame is useful in applications since in the flat limit (when $\omega_{\mathrm{H}} \rightarrow 0$ ) its coordinates become just the Cartesian ones of the Minkowski spacetime. In the frames 
with combined coordinates $\left\{t, \mathbf{x}_{\mathrm{c}}\right\}$ the metric takes the FLRW form

$\mathrm{d} s^{2}=\mathrm{d} t^{2}-a(t)^{2} \mathrm{~d} \mathbf{x}_{\mathrm{c}} \cdot \mathrm{d} \mathbf{x}_{\mathrm{c}}, \quad a(t)=e^{\omega_{\mathrm{H}} t}$,

where $a(t)$ is the scale factor of the expanding portion which can be rewritten in the conformal frame,

$a\left(t_{\mathrm{c}}\right) \equiv a\left[t\left(t_{\mathrm{c}}\right)\right]=-\frac{1}{\omega_{\mathrm{H}} t_{\mathrm{c}}}$,

as a function defined for $t_{\mathrm{c}}<0$.

The de Sitter spacetime is a hyperbolic manifold with the maximal symmetry whose isometry group is just the gauge group $S O(1,4)$ of the embedding manifold $M^{5}$ that leaves invariant its metric and implicitly Eq. (1). Therefore, given a system of coordinates defined by the functions $z=z(x)$, each transformation $\mathfrak{g} \in S O(1,4)$ defines an isometry, $x \rightarrow$ $x^{\prime}=\phi_{\mathfrak{g}}(x)$, derived from the system of equations

$z\left[\phi_{\mathfrak{g}}(x)\right]=\mathfrak{g} z(x)$.

The frames related through such isometries play the role of the inertial frames as in special relativity. Each isometry $x \rightarrow x^{\prime}=\phi_{\mathfrak{g}(\xi)}(x)$, depending on the group parameter $\xi$, gives rise to an associated Killing vector, $k=\left.\partial_{\xi} \phi_{\xi}\right|_{\xi=0}$. In a canonical parametrization of the $S O(1,4)$ group with real skew-symmetric parameters, $\xi^{A B}=-\xi^{B A}$, any infinitesimal isometry,

$\phi_{\mathfrak{g}(\xi)}^{\mu}(x)=x^{\mu}+\xi^{A B} k_{(A B)}^{\mu}(x)+\cdots$,

depends on the components

$k_{(A B) \mu}=z_{A} \partial_{\mu} z_{B}-z_{B} \partial_{\mu} z_{A}, \quad z_{A}=\eta_{A B} z^{B}$,

of the Killing vector associated to the parameter $\xi_{A B}$.

The classical conserved quantities along geodesics have the general form $\mathscr{K}_{(A B)}(x, \mathbf{P})=\omega_{\mathrm{H}} k_{(A B) \mu} p^{\mu}$ where the four-momentum components $p^{\mu}=\frac{\mathrm{d} x^{\mu}(s)}{\mathrm{d} \lambda}$ are the derivatives with respect to the affine parameter $\lambda$ which satisfies $d s=$ $m \mathrm{~d} \lambda$ such that $g_{\mu \nu} p^{\mu} p^{\nu}=m^{2}$. The conserved quantities with physical meaning [13] are the energy $E=\omega_{\mathrm{H}} k_{(04) \mu} p^{\mu}$, the angular momentum components, $L_{i}=\frac{1}{2} \varepsilon_{i j k} k_{(j k) \mu} p^{\mu}$, and the components $K_{i}=k_{(0 i)} \mu p^{\mu}$ and $R_{i}=k_{(i 4) \mu} p^{\mu}$ of two vectors related to the conserved momentum $\mathbf{P}$ and its associated dual momentum $\mathbf{Q}$ as, [13]

$\mathbf{P}=-\omega_{\mathrm{H}}(\mathbf{R}+\mathbf{K}), \quad \mathbf{Q}=\omega_{\mathrm{H}}(\mathbf{K}-\mathbf{R})$,

satisfying the identity

$E^{2}-\omega_{\mathrm{H}}^{2} \mathbf{L}^{2}-\mathbf{P} \cdot \mathbf{Q}=m^{2}$, corresponding to the first Casimir invariant of the $\operatorname{so}(1,4)$ algebra [13]. In the flat limit, $\omega_{\mathrm{H}} \rightarrow 0$ and $-\omega_{\mathrm{H}} t_{\mathrm{c}} \rightarrow 1$, we have $\mathbf{Q} \rightarrow \mathbf{P}$ such that this identity becomes just the usual mass-shell condition $E^{2}-\mathbf{P}^{2}=m^{2}$ of special relativity. The conserved quantities $\mathscr{K}_{(A B)}$ transform as a five-dimensional skew-symmetric tensor under the $S O(1,4)$ transformations generating the de Sitter isometries [6].

The simple isometries we meet here are the translations of parameters $\mathbf{d}=\left(d^{1}, d^{2}, d^{3}\right)$ that act as

$t_{\mathrm{c}}=t_{\mathrm{c}}^{\prime}, \quad \rightarrow \quad t=t^{\prime}$,

$\mathbf{x}_{\mathrm{c}}=\mathbf{x}_{\mathrm{c}}^{\prime}+\mathbf{d}, \rightarrow \mathbf{x}=\mathbf{x}^{\prime}+\mathbf{d} e^{\omega_{\mathrm{H}} t}$.

Hereby we can derive the Killing vector of components

$k_{j}^{0}=0, \quad k_{j}^{i}=\left.\frac{\partial x_{\mathrm{c}}^{i}}{\partial d^{j}}\right|_{\mathbf{d}=0}=\delta_{j}^{i}=\omega_{\mathrm{H}}\left(k_{(0, j)}^{i}+k_{(i, 4)}^{i}\right)$,

where $k_{(A B)}^{i}=g^{i j}\left(x_{\mathrm{c}}\right) k_{(A B) j}$ result from Eq. (10). This gives rise to the conserved momentum of the classical approach and to the momentum operator of the quantum theory. We shall see in the next section that these isometries transform the energy, angular momentum and dual momentum but preserve the conserved momentum.

\section{Null geodesics and redshift}

We consider now the null geodesics of the photons (with $m=0$ ) denoting the conserved quantities along these geodesics with capital letters. In the conformal frame the four-momentum components, denoted now by $k_{\mathrm{c}}^{\mu}=\frac{\mathrm{d} x_{\mathrm{c}}^{\mu}}{\mathrm{d} \lambda}$, satisfy the identity

$g_{\mu \nu}\left(x_{\mathrm{c}}\right) k_{\mathrm{c}}^{\mu} k_{\mathrm{c}}^{\nu}=a\left(t_{\mathrm{c}}\right)^{2}\left[k_{\mathrm{c}}^{0}\left(t_{\mathrm{c}}\right)^{2}-\mathbf{k}_{\mathrm{c}}\left(t_{\mathrm{c}}\right)^{2}\right]=0$,

resulting from the line element (3). In addition, we may consider the components

$P^{j}=-g_{j k}\left(x_{\mathrm{c}}\right) k_{i}^{j} \frac{\mathrm{d} x_{\mathrm{c}}^{k}}{\mathrm{~d} \lambda}=a\left(t_{\mathrm{c}}\right)^{2} \frac{\mathrm{d} x_{\mathrm{c}}^{j}}{\mathrm{~d} \lambda}$,

of the conserved momentum $\mathbf{P}=\mathbf{n}_{P} P(P=|\mathbf{P}|)$. Then by using the prime integrals (15) and (16) we derive the energy and covariant momentum in this frame as

$k_{\mathrm{c}}^{0}\left(t_{\mathrm{c}}\right)=\frac{\mathrm{d} t_{\mathrm{c}}}{\mathrm{d} \lambda}=\frac{P}{a\left(t_{\mathrm{c}}\right)^{2}}=\omega_{\mathrm{H}}^{2} t_{\mathrm{c}}^{2} P$,

$\mathbf{k}_{\mathrm{c}}\left(t_{\mathrm{c}}\right)=\frac{\mathrm{d} \mathbf{x}_{\mathrm{c}}}{\mathrm{d} \lambda}=\frac{\mathbf{P}}{a\left(t_{\mathrm{c}}\right)^{2}}=\omega_{\mathrm{H}}^{2} t_{\mathrm{c}}^{2} \mathbf{P}$.

The null geodesic results simply as [19]

$\mathbf{x}_{\mathrm{c}}\left(t_{\mathrm{c}}\right)=\mathbf{x}_{\mathrm{c} 0}+\mathbf{n}_{P}\left(t_{\mathrm{c}}-t_{\mathrm{c} 0}\right)$, 
leading us to conclude that this is determined completely by the unit vector $\mathbf{n}_{P}$ and the initial condition $\mathbf{x}_{\mathrm{c}}\left(t_{\mathrm{c} 0}\right)=\mathbf{x}_{\mathrm{c} 0}$.

The corresponding physical quantities measured in the frame $\{t, \mathbf{x}\}$ may be obtained by substituting the physical coordinates according to Eq. (4). Thus we find

$$
\begin{aligned}
k^{0}(t) & =\frac{\mathrm{d} t}{\mathrm{~d} \lambda}=P e^{-\omega_{\mathrm{H}} t}, \\
\mathbf{k}(t) & =\frac{\mathrm{d} \mathbf{x}}{\mathrm{d} \lambda}=\mathbf{P} e^{-\omega_{\mathrm{H}} t}+\omega_{\mathrm{H}} \mathbf{x}(t) P e^{-\omega_{\mathrm{H}} t},
\end{aligned}
$$

which represent the measured energy and covariant momentum in the point $[t, \mathbf{x}(t)]$ of the null geodesic [19]

$\mathbf{x}(t)=\mathbf{x}_{0} e^{\omega_{\mathrm{H}}\left(t-t_{0}\right)}+\mathbf{n}_{P} \frac{e^{\omega_{\mathrm{H}}\left(t-t_{0}\right)}-1}{\omega_{\mathrm{H}}}$,

which is passing through the space point $\mathbf{x}\left(t_{0}\right)=\mathbf{x}_{0}$ at the initial time $t_{0}$. The conserved quantities on this geodesic can be calculated at any time as

$E=e^{-\omega_{\mathrm{H}} t}\left[P+\omega_{\mathrm{H}} \mathbf{x}(t) \cdot \mathbf{P}\right]$,

$\mathbf{L}=\mathbf{x}(t) \wedge \mathbf{P} e^{-\omega_{\mathrm{H}} t}$,

$\mathbf{Q}=2 \omega_{\mathrm{H}} \mathbf{x}(t) e^{-\omega_{\mathrm{H}} t} E+\mathbf{P} e^{-2 \omega_{\mathrm{H}} t}\left[1-\omega_{\mathrm{H}}^{2} \mathbf{x}(t)^{2}\right]$,

observing that these satisfy the identity (12) for $m=0$.

The momentum defined by Eq. (21) can be split as $\mathbf{k}(t)=$ $\hat{\mathbf{k}}(t)+\overline{\mathbf{k}}(t)$ where

$\hat{\mathbf{k}}(t)=\mathbf{P} e^{-\omega_{\mathrm{H}} t}, \quad \overline{\mathbf{k}}(t)=\omega_{\mathrm{H}} \mathbf{x}(t) k^{0}(t)$,

are the peculiar and recessional momenta we have defined recently [20]. The prime integral derived from the line element (5) gives the familiar identity

$k^{0}(t)^{2}-\hat{\mathbf{k}}(t)^{2}=0$,

which is just the mass-shell condition of special relativity satisfied by the energy and peculiar momentum along the null geodesics.

Now we may analyze how two different observers measure a photon moving on a null geodesic which is passing through the origins $O$ and $O^{\prime}$ of their proper physical frames $\{t, \mathbf{x}\}_{O}$ and $\left\{t, \mathbf{x}^{\prime}\right\}_{O^{\prime}}$. We assume that the photon is emitted in $O^{\prime}$ at the initial time $t_{0}$ when the origin $O^{\prime}$ is translated with respect to $O$ as

$\mathbf{x}\left(t_{0}\right)=\mathbf{x}^{\prime}\left(t_{0}\right)+\mathbf{d} e^{\omega_{\mathrm{H}} t_{0}}$,

where the translation parameter $\mathbf{d}=\mathbf{n} d$ of the isometry (13) has the direction $O O^{\prime}$ given by the unit vector $\mathbf{n}$. If we know that the photon is emitted in $\mathbf{x}^{\prime}\left(t_{0}\right)=0$ with the momentum $\mathbf{k}=-\mathbf{n} k$ and energy $k^{0}=k$ we may ask what the energy and momentum are of this photon measured in the origin $O$ at the final time $t_{f}$ when the photon reaches this point. For solving this problem we look first for the conserved momentum that is the same in the points $O^{\prime}$ and $O$,

$\mathbf{P}^{\prime}=\mathbf{P}=\mathbf{k} e^{\omega_{M} t_{0}} \rightarrow P=k e^{\omega_{M} t_{0}}, \quad \mathbf{n}_{P}=-\mathbf{n}$,

since this is invariant under translations being associated to their generators. Furthermore, we observe that the choice of the initial time

$t_{\mathrm{c} 0}=-\frac{1}{\omega_{\mathrm{H}}} \rightarrow t_{0}=0$

when $a=1$ and, consequently, the conformal and physical space coordinates coincide. This simplifies the calculations, allowing us to find the quantities measured by the observers $O$ and $O^{\prime}$ at this moment derived from Eqs. (23)-(25).

The results are presented in the next table where we introduce the intuitive notations for the initial, $E_{i}$, and final, $E_{f}$, photon energies which are the physical quantities involved in the redshift. We have

$$
\text { frame }\left\{t, \mathbf{x}^{\prime}\right\}_{O^{\prime}} \text { frame }\{t, \mathbf{x}\}_{O},
$$

initial cond. $\quad \mathbf{x}^{\prime}(0)=0 \quad \mathbf{x}(0)=\mathbf{d}$,

cons. energy $\quad E^{\prime} \equiv E_{i}=k \quad E \equiv E_{f}=k\left(1-\omega_{\mathrm{H}} d\right)$,

cons. mom. $\quad \mathbf{P}^{\prime}=\mathbf{k} \quad \mathbf{P}=\mathbf{k}$,

angular mom. $\mathbf{L}^{\prime}=0 \quad \mathbf{L}=0$,

dual mom. $\quad \mathbf{Q}^{\prime}=\mathbf{k} \quad \mathbf{Q}=\mathbf{k}\left(1-\omega_{\mathrm{H}} d\right)^{2}$.

We observe that the translation (28) changes the energy and the components of the adjoint momentum but preserving the invariant (12) with $m=0$. Indeed, in the frame $\left\{t, \mathbf{x}^{\prime}\right\}_{O^{\prime}}$ we have $E_{i}^{2}=\mathbf{k}^{2}=\mathbf{P}^{\prime} \cdot \mathbf{Q}^{\prime}$. In the frame $\{t, \mathbf{x}\}_{O}$ the photon arrives in $\mathbf{x}=0$ at the conformal time $t_{\mathrm{cf}}=d-\frac{1}{\omega_{\mathrm{H}}}$ corresponding to the cosmic time

$t_{f}=-\frac{1}{\omega_{\mathrm{H}}} \ln \left(-\omega_{\mathrm{H}} t_{\mathrm{cf}}\right)=-\frac{1}{\omega_{\mathrm{H}}} \ln \left(1-\omega_{\mathrm{H}} d\right)$.

Hereby we can deduce that $O$ measures the final peculiar momentum,

$\hat{\mathbf{k}}\left(t_{f}\right)=\mathbf{k}\left(1-\omega_{\mathrm{H}} d\right)$

resulting from Eq. (26) for $t=t_{f}$. Obviously, this satisfies the mass-shell condition $E_{f}^{2}=\hat{\mathbf{k}}\left(t_{f}\right)^{2}=\mathbf{P} \cdot \mathbf{Q}$. Hereby we conclude that $\mathbf{P}$ and $\mathbf{Q}$ are conserved quantities that cannot be measured directly but complete each other for closing the identity (12). The only measurable quantities remain thus the energy, peculiar momentum and angular momentum.

These results allow us to recover the Lemaittre expression of Hubble's law giving the redshift $z$ as

$\frac{1}{1+z}=\frac{E_{\mathrm{f}}}{E_{\mathrm{i}}}=1-\omega_{\mathrm{H}} d=1-\frac{d}{l_{\mathrm{H}}}$, 
and the physical observer-source distance at the time $t_{\mathrm{f}}$,

$d_{\mathrm{f}}=\frac{d}{-\omega_{\mathrm{H}} t_{\mathrm{cf}}}=\frac{d}{1-\omega_{\mathrm{H}} d}$,

which was increasing because of the space expansion during the photon propagation. Thus we revisited the redshift in the particular case when $O^{\prime}$ does not have a peculiar velocity. The general result for arbitrary peculiar velocity of $O^{\prime}$ was derived recently $[8,9]$.

These results that hold at the level of the geometric optics in de Sitter background are incomplete since at this level we neglect the wave behavior and polarization that may be studied considering the classical and quantum theory of the Maxwell field.

\section{Classical Maxwell field}

Let us consider first the classical approach denoting by $A$ the electromagnetic potential of the Maxwell field minimally coupled to the de Sitter gravity, whose action in an arbitrary frame $\{x\}$ of $M_{+}$reads

$\mathcal{S}[A]=\int \mathrm{d}^{4} x \sqrt{g} \mathscr{L}=-\frac{1}{4} \int \mathrm{d}^{4} x \sqrt{g} F_{\mu \nu} F^{\mu \nu}$,

where $g=\left|\operatorname{det}\left(g_{\mu \nu}\right)\right|$ and $F_{\mu \nu}=\partial_{\mu} A_{\nu}-\partial_{\nu} A_{\mu}$ is the field strength. From this action one derives the field equations

$\partial_{\nu}\left(\sqrt{g} g^{\nu \alpha} g^{\mu \beta} F_{\alpha \beta}\right)=0$,

which are invariant under conformal transformations, $g_{\mu \nu} \rightarrow$ $g_{\mu \nu}^{\prime}=\Omega g_{\mu \nu}$ and

$A_{\mu} \rightarrow A_{\mu}^{\prime}=A_{\mu} \quad A^{\mu} \rightarrow A^{\prime \mu}=\Omega^{-1} A^{\mu}$.

The canonical variables $A_{\mu}$ must obey, in addition, the Lorentz condition

$\partial_{\mu}\left(\sqrt{g} g^{\mu v} A_{\nu}\right)=0$

which is no longer conformally invariant since

$\partial_{\mu}\left(\sqrt{g^{\prime}} g^{\prime \mu v} A_{v}^{\prime}\right)=\partial_{\mu}\left(\sqrt{g} g^{\mu v} A_{v}\right)+\sqrt{g} A^{\mu} \partial_{\mu} \Omega$.

However, we may get over this inconvenience imposing the Coulomb gauge, $A_{0}=0$, in the conformal frame $\left\{t_{\mathrm{c}}, \mathbf{x}_{\mathrm{c}}\right\}$, since then the second term of Eq. (39) does not contribute.

\subsection{Plane waves}

Under such circumstances we can write the solutions of the Maxwell equations in Coulomb gauge, $\left(\partial_{t_{\mathrm{c}}}^{2}-\Delta_{\mathrm{c}}\right) A_{i}=0$, and the Lorentz condition, $\partial_{x_{\mathrm{c}}^{i}} A_{i}=0$, taking over all the wellknown results of special relativity. Thus we get the plane wave solutions

$$
\begin{aligned}
& A_{i}\left(x_{\mathrm{c}}\right)=A_{i}^{(+)}\left(x_{\mathrm{c}}\right)+A_{i}^{(-)}\left(x_{\mathrm{c}}\right) \\
& =\int \mathrm{d}^{3} k \sum_{\lambda}\left[e_{i}\left(\mathbf{n}_{k}, \lambda\right) \hat{f}_{\mathbf{k}}\left(x_{\mathrm{c}}\right) \hat{a}(\mathbf{k}, \lambda)\right. \\
& \left.+\left[e_{i}\left(\mathbf{n}_{k}, \lambda\right) \hat{f}_{\mathbf{k}}\left(x_{\mathrm{c}}\right)\right]^{*} \hat{a}^{*}(\mathbf{k}, \lambda)\right],
\end{aligned}
$$

expressed in terms of wave functions in momentum representation, $\hat{a}(\mathbf{k}, \lambda)$, polarization vectors, $e_{i}\left(\mathbf{n}_{k}, \lambda\right)$, and fundamental solutions of the d'Alembert equation,

$\hat{f}_{\mathbf{k}}\left(x_{\mathrm{c}}\right)=\frac{1}{(2 \pi)^{3 / 2}} \frac{1}{\sqrt{2 k}} e^{i \delta(\mathbf{k})-i k t_{\mathrm{c}}+i \mathbf{k} \cdot \mathbf{x}_{\mathrm{c}}}$,

where $\mathbf{k}=k \mathbf{n}_{k}$ is the momentum vector with $k=|\mathbf{k}|$.

The momentum-dependent phase $\delta(\mathbf{k})$ is introduced in order to ensure the correct flat limit of the plane wave solutions when $\omega_{\mathrm{H}} \rightarrow 0$. According to Eq. (4), we see that the entire phase of the function (41) behaves as

$i \delta(\mathbf{k})-i k\left(-\frac{1}{\omega_{\mathrm{H}}}+t\right)+i \mathbf{x}_{\mathrm{c}} \cdot \mathbf{k}+\mathscr{O}\left(\omega_{\mathrm{H}}\right)$,

having a pole in $\omega_{\mathrm{H}}=0$. For removing this singularity we must impose the condition

$\lim _{\omega_{\mathrm{H}} \rightarrow 0}\left(\delta(\mathbf{k})+\frac{k}{\omega_{\mathrm{H}}}\right)=0$,

giving the correct flat limit of special relativity,

$\lim _{\omega_{\mathrm{H}} \rightarrow 0} e^{i \delta(\mathbf{k})-i k t_{\mathrm{c}}+i \mathbf{k} \cdot \mathbf{x}_{\mathrm{c}}}=e^{-i k t+i \mathbf{k} \cdot \mathbf{x}}$.

For avoiding some difficulties related to this explicit phase, it is convenient to redefine

$$
f_{\mathbf{k}}\left(x_{\mathrm{c}}\right)=e^{-i \delta(\mathbf{k})} \hat{f}_{\mathbf{k}}\left(x_{\mathrm{c}}\right)=\frac{1}{(2 \pi)^{3 / 2}} \frac{1}{\sqrt{2 k}} e^{-i k t_{\mathrm{c}}+i \mathbf{k} \cdot \mathbf{x}_{\mathrm{c}}},
$$

$a(\mathbf{k}, \lambda)=e^{i \delta(\mathbf{k})} \hat{a}(\mathbf{k}, \lambda)$,

substituting $\hat{f}_{\mathbf{k}}\left(x_{\mathrm{c}}\right) \hat{a}(\mathbf{k}, \lambda)=f_{\mathbf{k}}\left(x_{\mathrm{c}}\right) a(\mathbf{k}, \lambda)$ in Eq. (40).

In Ref. [11] we neglected the problem of this phase which is solved here for the first time. This guarantees the correct flat limit as in the case of the rest frame vacua we defined recently for the massive Dirac [21], Klein-Gordon [22] and Proca [23] free fields, showing that only these vacua ensure correct flat limits [24]. We specify that the covariant KleinGordon and Dirac free fields on the de Sitter manifold were studied for the first time in Ref. [25] which was the starting point in obtaining our mentioned results. 
The functions $f_{\mathbf{k}}(x)$ are assumed to be of positive frequencies while those of negative frequencies are $f_{\mathbf{k}}(x)^{*}$. These solutions satisfy the orthonormalization relations [11]

$$
\begin{aligned}
\left(f_{\mathbf{k}}, f_{\mathbf{k}^{\prime}}\right)=-\left(f_{\mathbf{k}}^{*}, f_{\mathbf{k}^{\prime}}^{*}\right) & =\delta^{3}\left(\mathbf{k}-\mathbf{k}^{\prime}\right), \\
\left(f_{\mathbf{k}}, f_{\mathbf{k}^{\prime}}^{*}\right) & =0,
\end{aligned}
$$

and the completeness condition

$i \int \mathrm{d}^{3} k f_{\mathbf{k}}^{*}\left(t_{\mathrm{c}}, \mathbf{x}_{\mathrm{c}}\right) \stackrel{\leftrightarrow}{\partial_{t_{\mathrm{c}}}} f_{\mathbf{k}}\left(t_{\mathrm{c}}, \mathbf{x}^{\prime}\right)=\delta^{3}\left(\mathbf{x}_{\mathrm{c}}-\mathbf{x}_{\mathrm{c}}^{\prime}\right)$

with respect to the Hermitian form

$(f, g)=i \int \mathrm{d}^{3} x_{\mathrm{c}} f^{*}\left(t_{\mathrm{c}}, \mathbf{x}_{\mathrm{c}}\right) \stackrel{\leftrightarrow}{\partial_{t_{\mathrm{c}}}} g\left(t_{\mathrm{c}}, \mathbf{x}_{\mathrm{c}}\right)$

Here we denote $f \stackrel{\leftrightarrow}{\partial} g=f \partial g-g \partial f$.

The polarization vectors $\mathbf{e}\left(\mathbf{n}_{k}, \lambda\right)$ in Coulomb gauge must be orthogonal to the momentum direction,

$\mathbf{k} \cdot \mathbf{e}\left(\mathbf{n}_{k}, \lambda\right)=0$,

for any polarization $\lambda= \pm 1$. We remind the reader that the polarization can be defined in different manners independent of the form of the scalar solutions $f_{\mathbf{k}}$. In general, the polarization vectors have c-number components which must satisfy [26]

$$
\begin{aligned}
\mathbf{e}\left(\mathbf{n}_{k}, \lambda\right) \cdot \mathbf{e}\left(\mathbf{n}_{k}, \lambda^{\prime}\right)^{*} & =\delta_{\lambda \lambda^{\prime}}, \\
\sum_{\lambda} e_{i}\left(\mathbf{n}_{k}, \lambda\right) e_{j}\left(\mathbf{n}_{k}, \lambda\right)^{*} & =\delta_{i j}-\frac{k^{i} k^{j}}{k^{2}} .
\end{aligned}
$$

Here we restrict ourselves to considering only the circular polarization for which the supplementary condition $\mathbf{e}\left(\mathbf{n}_{k}, \lambda\right)^{*} \wedge \mathbf{e}\left(\mathbf{n}_{k}, \lambda\right)=i \lambda \mathbf{n}_{k}$ is required.

We obtained thus the mode expansions in terms of transverse plane waves of given momentum and helicity. The functions $w_{i(\mathbf{k}, \lambda)}=e_{i}\left(\mathbf{n}_{k}, \lambda\right) f_{\mathbf{k}}$, are of positive frequencies while those of negative frequencies are $w_{i(\mathbf{k}, \lambda)}^{*}$. We say that these sets of fundamental solutions of the Maxwell equation define the momentum-helicity basis. Note that an energy-helicity basis can also be defined [11].

\subsection{Conserved quantities}

The Maxwell theory is invariant under $S O(1,4)$ isometries such that under a given isometry, $x \rightarrow x^{\prime}=\phi_{\mathfrak{g}(\xi)}(x)$, depending on the group parameter $\xi$, the vector field $A$ transforms as $A \rightarrow A^{\prime}=T_{\xi} A$, according to the operator-valued representation $\xi \rightarrow T_{\xi}$ of the isometry group defined by the well-known rule

$\frac{\partial \phi_{\xi}^{\nu}(x)}{\partial x_{\mu}}\left(T_{\xi} A\right)_{\nu}[\phi(x)]=A_{\mu}(x)$.
The corresponding generator, $X_{K}=\left.i \partial_{\xi} T_{\xi}\right|_{\xi=0}$, has the action

$\left(X_{k} A\right)_{\mu}=-i\left(k^{\nu} A_{\mu ; \nu}+k_{; \mu}^{\nu} A_{\nu}\right)$,

where $k$ is the Killing vector associated to $\xi$. In a canonical parametrization of the $S O(1,4)$ group we have the correspondence

$\xi^{A B} \rightarrow k_{(A B)} \rightarrow X_{k_{(A B)}} \equiv X_{(A B)}$,

which means that the generators $X_{(A B)}$ form a basis of the vector representation of the $s o(4,1)$ algebra carried by the space of the vector potential, $A$. This algebra yields the principal observables, i.e. the energy operator $\hat{H}=\omega X_{(05)}$, the components of the momentum, $\hat{P}^{i}=-\omega_{\mathrm{H}}\left(X_{(i 4)}+X_{(0 i)}\right)$, dual momentum, $\hat{Q}^{i}=\omega_{\mathrm{H}}\left(X_{(0 i)}-X_{(i 4)}\right)$, and total angular momentum, $\hat{J}_{i}=\frac{1}{2} \varepsilon_{i j k} X_{(j k)}(i, j, \ldots=1,2,3)$, operators $[13,27]$.

The action of these operators can be calculated according to Eq. (55) using the concrete form of the Killing vectors derived from Eq. (10). In what follows we restrict ourselves to the results in the Coulomb gauge (with $A_{0}=0$ ) which are useful in applications. The energy and momentum operators do not have spin parts, acting as [11]

$$
\begin{aligned}
& \hat{H} A_{j}=-i \omega_{\mathrm{H}}\left(t_{\mathrm{c}} \frac{\partial}{\partial t_{\mathrm{c}}}+x_{\mathrm{c}}^{i} \frac{\partial}{\partial x_{\mathrm{c}}^{i}}+1\right) A_{j}, \\
& \hat{P}^{i} A_{j}=-i \frac{\partial}{\partial x_{\mathrm{c}}^{i}} A_{j},
\end{aligned}
$$

while the action of the total angular momentum reads

$\hat{J}_{i} A_{j}=\hat{L}_{i} A_{j}-i \varepsilon_{i j k} A_{k}$,

where $\hat{\mathbf{L}}=\mathbf{x}_{\mathrm{c}} \times \mathbf{P}$ is the usual angular momentum operator. In addition, we define the Pauli-Lubanski (or helicity) operator $\hat{W}=\hat{\mathbf{P}} \cdot \hat{\mathbf{J}}$ whose action depends only on the spin parts,

$\hat{W} A_{i}=\varepsilon_{i j k} \frac{\partial}{\partial x_{\mathrm{c}}^{j}} A_{k}$.

This operator will define the polarization in the canonical basis of the so(3) algebra as in special relativity [11]. In contrast with these operators, the dual momentum has the more complicated action,

$$
\begin{aligned}
\hat{Q}^{i} A_{j}= & -i \omega_{\mathrm{H}}^{2}\left[2 x_{\mathrm{c}}^{i}\left(t_{\mathrm{c}} \frac{\partial}{\partial t_{\mathrm{c}}}+x_{\mathrm{c}}^{k} \frac{\partial}{\partial x_{\mathrm{c}}^{k}}\right)+\left(t_{\mathrm{c}}^{2}-\mathbf{x}_{\mathrm{c}}^{2}\right) \frac{\partial}{\partial x_{\mathrm{c}}^{i}}\right] A_{j} \\
& -i \omega_{\mathrm{H}}^{2}\left(2 \delta_{i j} \mathbf{x}_{\mathrm{c}} \cdot \mathbf{A}+x_{\mathrm{c}}^{i} A_{j}-x_{\mathrm{c}}^{j} A_{i}\right),
\end{aligned}
$$

we present here for the first time.

We constructed thus the basis $\left\{\hat{H}, \hat{P}^{i}, \hat{Q}^{i}, \hat{J}_{i}\right\}$ of the vector representation of the $s o(1,4)$ algebra whose commutation rules are 


$$
\begin{aligned}
& {\left[\hat{H}, \hat{J}_{i}\right]=0} \\
& {\left[\hat{H}, \hat{P}^{i}\right]=i \omega_{\mathrm{H}} \hat{P}^{i}, \quad\left[\hat{H}, \hat{Q}^{i}\right]=-i \omega_{\mathrm{H}} \hat{Q}^{i},} \\
& {\left[\hat{J}_{i}, \hat{P}^{j}\right]=i \varepsilon_{i j k} \hat{P}^{k}, \quad\left[\hat{J}_{i}, \hat{Q}^{j}\right]=i \varepsilon_{i j k} \hat{Q}^{k},} \\
& {\left[\hat{Q}^{i}, \hat{Q}^{j}\right]=0, \quad\left[\hat{P}^{i}, \hat{P}^{j}\right]=0,} \\
& {\left[\hat{Q}^{i}, \hat{P}^{j}\right]=2 i \omega_{\mathrm{H}} \delta_{i j} \hat{H}+2 i \omega_{\mathrm{H}}^{2} \varepsilon_{i j k} \hat{J}_{k},}
\end{aligned}
$$

from which we deduce

$\left[\hat{P}^{i}, \hat{W}\right]=0, \quad[\hat{H}, \hat{W}]=i \omega_{\mathrm{H}} \hat{W}$,

understanding that the maximal set of commuting operators we may use is $\left\{\hat{P}^{i}, \hat{W}\right\}$. The first Casimir operator of this algebra has the form [13]

$\mathscr{C}_{1}=\hat{H}^{2}+3 i \omega_{\mathrm{H}} \hat{H}-\hat{\mathbf{Q}} \cdot \hat{\mathbf{P}}-\omega_{\mathrm{H}}^{2} \hat{\mathbf{J}} \cdot \hat{\mathbf{J}}$

giving the supplemental equation $\mathscr{C}_{1} A_{i}=0$ which in the Coulomb gauge is just the d'Alembert one.

In what concerns the structure of the $s o(1,4)$ algebra we observe that there are two Abelian sub-algebras generated by the momentum components, $\left\{\hat{P}^{i}\right\}$, respectively, by those of the dual momentum, $\left\{\hat{Q}^{i}\right\}$. Another specific feature is that the energy operators does not commute with the momentum components as in special relativity. Regarding our notations we must specify that the upper or lower positions of the space indices do not have here a meaning but we denoted the components of the momentum and dual momentum with upper indices since in the flat limit these become $\hat{Q}^{i} \rightarrow \hat{P}^{i} \rightarrow-i \partial_{i}$, i.e. the contravariant space components of the momentum operator with respect to the Minkowski metric.

The conserved quantities of our Lagrangian theory are related to the operators of the $s o(1,4)$ algebra via Noether's theorem. From the action (35) we deduce that in Coulomb's gauge the conserved quantities can be derived by using the Hermitian form (50) as [11]

$$
X \rightarrow C[X]=\frac{1}{2} \delta_{i j}\left(A_{i}, X A_{j}\right) \quad \forall X \in \operatorname{so}(1,4) .
$$

This integral can be expressed in terms of electric and magnetic components of the field strength [12] or as a mode integral in momentum representation. Thus we can conclude that we outlined here a coherent de Sitter electrodynamics which has a correct flat limit.

\section{Quantum Maxwell field}

The next step is the quantization we may perform in a canonical manner as in special relativity exploiting the global con- formal invariance of the theory in Coulomb gauge. There are many delicate problems that can be avoided if we restrict ourselves to the conserved operators in the Heisenberg picture assuming that the quantum states are defined at the initial time (30) when $a(0)=1$ and the conserved and peculiar momenta coincide. Then we have to focus only on the conserved operators calculated at this moment which are enough for analyzing the quantum redshift. However, this method is not suitable for studying other dynamic operators as, for example, the coordinate operator and the peculiar momentum one.

\subsection{One-particle operators}

We assume that the wave functions $a$ of the field (40) become field operators (with $a^{*} \rightarrow a^{\dagger}$ ) [26] such that the potentials (40) become field operators denoted by $\mathscr{A}_{i}$. We assume that the field operators fulfill the standard commutation relations in the momentum-helicity basis from which the nonvanishing ones are

$\left[a(\mathbf{k}, \lambda), a^{\dagger}\left(\mathbf{k}^{\prime}, \lambda^{\prime}\right)\right]=\delta_{\lambda \lambda^{\prime}} \delta^{3}\left(\mathbf{k}-\mathbf{k}^{\prime}\right)$.

Then the Hermitian field $\mathscr{A}=\mathscr{A}^{\dagger}$ is correctly quantized according to the canonical rule

$$
\begin{aligned}
{\left[\mathscr{A}_{i}\left(t_{\mathrm{c}}, \mathbf{x}_{\mathrm{c}}\right), \pi^{j}\left(t_{\mathrm{c}}, \mathbf{x}_{\mathrm{c}}^{\prime}\right)\right] } & =\left[\mathscr{A}_{i}\left(t_{\mathrm{c}}, \mathbf{x}\right), \partial_{t_{\mathrm{c}}} \mathscr{A}_{j}\left(t_{\mathrm{c}}, \mathbf{x}_{\mathrm{c}}^{\prime}\right)\right] \\
& =i \delta_{i j}^{t r}\left(\mathbf{x}_{\mathrm{c}}-\mathbf{x}_{\mathrm{c}}^{\prime}\right),
\end{aligned}
$$

where

$\pi^{j}=\sqrt{g} \frac{\delta \mathscr{L}}{\delta\left(\partial_{t_{\mathrm{c}}} \mathscr{A}_{j}\right)}=\partial_{t_{\mathrm{c}}} \mathscr{A}_{j}$

is the momentum density in Coulomb gauge $\left(\mathscr{A}_{0}=0\right)$ and

$\delta_{i j}^{t r}\left(\mathbf{x}_{\mathrm{c}}\right)=\frac{1}{(2 \pi)^{3}} \int \mathrm{d}^{3} q\left(\delta_{i j}-\frac{q^{i} q^{j}}{q^{2}}\right) e^{i \mathbf{q} \cdot \mathbf{x}_{\mathrm{c}}}$

is the well-known transverse $\delta$-function [26] arising from Eq. (53).

As in special relativity we consider a unique vacuum state, $|0\rangle$, of the Fock space such that

$a(\mathbf{k}, \lambda)|0\rangle=0, \quad\langle 0| a^{\dagger}(\mathbf{k}, \lambda)=0$.

The sectors with a given number of particles may be constructed using the standard methods for obtaining the generalized momentum-helicity basis of the Fock space.

The one-particle operators corresponding to the conserved quantities (69) can be calculated in Coulomb gauge as [11]

$\mathscr{X}=\frac{1}{2} \delta_{i j}:\left(\mathscr{A}_{i}, X \mathscr{A}_{j}\right)$, 
respecting the normal ordering of the operator products [26]. The obvious algebraic properties

$$
\begin{aligned}
{\left[\mathscr{X}, \mathscr{A}_{i}(x)\right] } & =-X \mathscr{A}_{i}(x), \\
{[\mathscr{X}, \mathscr{Y}] } & =\frac{1}{2} \delta_{i j}:\left(\mathscr{A}_{i},[X, Y] \mathscr{A}_{j}\right),
\end{aligned}
$$

are due to the canonical quantization adopted here. However, there are many other conserved operators which do not have corresponding differential operators but can be defined directly as mode expansions. The simplest example is the operator of the number of particles,

$\mathscr{N}=\int \mathrm{d}^{3} k \sum_{\lambda} a^{\dagger}(\mathbf{k}, \lambda) a(\mathbf{k}, \lambda)$.

The conserved one-particle operators whose mode expansions can be derived easily are the components of the conserved momentum operator,

$$
\mathscr{P}^{l}=\frac{1}{2} \delta_{i j}:\left(\mathscr{A}_{i}, \hat{P}^{l} \mathscr{A}_{j}\right)=\int \mathrm{d}^{3} k k^{l} \sum_{\lambda} a^{\dagger}(\mathbf{k}, \lambda) a(\mathbf{k}, \lambda),
$$

and the Pauli-Lubanski operator,

$$
\mathscr{W}=\frac{1}{2} \delta_{i j}:\left(\mathscr{A}_{i}, \hat{W} \mathscr{A}_{j}\right)=\int \mathrm{d}^{3} k k \sum_{\lambda} \lambda a^{\dagger}(\mathbf{k}, \lambda) a(\mathbf{k}, \lambda),
$$

which commutes with the momentum components, $\left[\mathscr{P}^{i}, \mathscr{W}\right]$ $=0$. The momentum-helicity basis,

$$
\begin{aligned}
& |0\rangle,|\mathbf{k}, \lambda\rangle=a^{\dagger}(\mathbf{k}, \lambda)|0\rangle, \\
& \left|\mathbf{k}, \lambda ; \mathbf{k}^{\prime}, \lambda^{\prime}\right\rangle=a^{\dagger}(\mathbf{k}, \lambda) a^{\dagger}\left(\mathbf{k}^{\prime}, \lambda^{\prime}\right)|0\rangle, \ldots
\end{aligned}
$$

is formed by the eigenvectors of the set of commuting operators $\left\{\mathscr{W}, \mathscr{P}^{i}\right\}$ corresponding to the discrete polarizations, $\lambda, \lambda+\lambda^{\prime}, \ldots \in \mathbb{Z}$ and momenta $\mathbf{k}, \mathbf{k}+\mathbf{k}^{\prime}, \ldots$ of the continuous spectrum $\mathbb{R}_{k}^{3}$.

The problem of the energy operator is more delicate but can be solved resorting to the identity

$\left(\hat{H} f_{\mathbf{k}}\right)(x)=-i \omega_{\mathrm{H}}\left(k^{i} \partial_{k_{i}}+\frac{3}{2}\right) f_{\mathbf{k}}(x)$,

satisfied by the functions (45). Then, after a few manipulations and applying the Green theorem, we obtain the final result [11],

$$
\begin{aligned}
\mathscr{H} & =\frac{1}{2} \delta_{i j}:\left(\mathscr{A}_{i}, \hat{H} \mathscr{A}_{j}\right): \\
& =\frac{i \omega_{\mathrm{H}}}{2} \int \mathrm{d}^{3} k k^{i} \sum_{\lambda} a^{\dagger}(\mathbf{k}, \lambda) \stackrel{\leftrightarrow}{\partial}_{k_{i}} a(\mathbf{k}, \lambda) .
\end{aligned}
$$

Hereby we see that the form of the energy operator is strongly dependent on the phases of the operators $a(\mathbf{k}, \lambda)$ defined by Eq. (46). This is in accordance with the similar property of the energy operator that holds for the Klein-Gordon, Dirac or Proca free fields on this background. This general behavior is due to the space expansion giving the dependence of the energy operator on the translations that change the phases. Nevertheless, this behavior does not change the commutation relations

$$
\begin{aligned}
{\left[\mathscr{H}, \mathscr{P}^{i}\right] } & =i \omega_{\mathrm{H}} \mathscr{P}^{i}, \\
{[\mathscr{H}, \mathscr{W}] } & =i \omega_{\mathrm{H}} \mathscr{W},
\end{aligned}
$$

which are independent on the phase $\delta(\mathbf{k})$ as they result from Eqs. (63) and (77).

\subsection{Wave packets and measurements}

A simple model which prevents us from complicated calculations is that of the one-particle wave packets. In our Heisenberg picture these are given by the time-independent one-particle states,

$|\alpha\rangle=\int \mathrm{d}^{3} k \sum_{\lambda} \alpha_{\lambda}(\mathbf{k}) a^{\dagger}(\mathbf{k}, \lambda)|0\rangle$,

defined by the square integrable functions in momentum representation $\alpha_{\lambda}(\mathbf{k})$ which must satisfy the normalization condition

$\langle\alpha \mid \alpha\rangle=\int \mathrm{d}^{3} k \sum_{\lambda}\left|\alpha_{\lambda}(\mathbf{k})\right|^{2}=1$.

The corresponding 'wave functions',

$A[\alpha]_{i}(x)=\left\langle 0\left|\mathscr{A}_{i}(x)\right| \alpha\right\rangle=\int \mathrm{d}^{3} k \sum_{\lambda} e_{i}\left(\mathbf{n}_{k}, \lambda\right) f_{\mathbf{k}}(x) \alpha_{\lambda}(\mathbf{k})$,

are known as wave packets. These are useful auxiliary functions related to those of the momentum representation through the inversion relations

$\alpha_{\lambda}(\mathbf{k})=\delta_{i j} e_{i}\left(\mathbf{n}_{k}, \lambda\right)^{*}\left(f_{\mathbf{k}}, A[\alpha]_{j}\right)$.

Moreover, the expectation values of the one-particle operators (75) in the state $|\alpha\rangle$ can be calculated simply as

$\langle\alpha|\mathscr{X}| \alpha\rangle=\delta_{i j}\left(A[\alpha]_{i}, X A[\alpha]_{j}\right)$,

avoiding the tedious algebra of field operators.

Once the wave packet is prepared this evolves causally until an ideal apparatus measures some of its parameters. 
More specific, this apparatus can measure all the eigenvalues of the operators $\mathscr{W}$ and $\mathscr{P}^{i}$ which are diagonal in the momentum-helicity basis. In an experiment we can set this apparatus to select only the momenta included in a desired domain $\Delta \subset \mathbb{R}_{k}^{3}$ by using a suitable projection operator, $\Lambda_{\Delta}=\Lambda_{\Delta}^{\dagger}$ (satisfying $\Lambda_{\Delta}^{2}=\Lambda_{\Delta}$ ), which can be represented as

$$
\Lambda_{\Delta}=|0\rangle\left\langle 0\left|+\int_{\Delta} \mathrm{d}^{3} k \sum_{\lambda} a^{\dagger}(\mathbf{k}, \lambda)\right| 0\right\rangle\langle 0| a(\mathbf{k}, \lambda)+\cdots,
$$

where the integral is restricted to the domain $\Delta$. During the experiment this operator filters only the momenta $\mathbf{k} \in \Delta$ transforming the state of the system into the new one $|\alpha\rangle \rightarrow$ $\Lambda_{\Delta}|\alpha\rangle$. Then the expectation value $\langle\mathscr{X}\rangle$ of a one-particle operator $\mathscr{X}$ can be calculated as [28]

$\langle\mathscr{X}\rangle=\frac{\left\langle\alpha\left|\Lambda_{\Delta} \mathscr{X}\right| \alpha\right\rangle}{\left\langle\alpha\left|\Lambda_{\Delta}\right| \alpha\right\rangle}$,

taking into account that our one-particle operators commute with $\Lambda_{\Delta}$. The quantity

$$
\left\langle\alpha\left|\Lambda_{\Delta}\right| \alpha\right\rangle=\int_{\Delta} \mathrm{d}^{3} k \sum_{\lambda}\left|\alpha_{\lambda}(\mathbf{k})\right|^{2} \leq 1
$$

gives the probability $P_{\Delta}=\left|\left\langle\alpha\left|\Lambda_{\Delta}\right| \alpha\right\rangle\right|^{2}$ of measuring any momentum $\mathbf{k} \in \Delta$. Obviously, when we can measure the whole continuous spectrum, $\Delta=\mathbb{R}_{k}^{3}$, then $\Lambda_{\Delta} \rightarrow \mathbf{1}, P_{\Delta}=1$ and $\langle\mathscr{X}\rangle=\langle\alpha|\mathscr{X}| \alpha\rangle$.

Furthermore, bearing in mind the role of the phase factor in Eq. (46) we assume that the functions $\alpha_{\lambda}$ have the general form

$\alpha_{\lambda}(\mathbf{k})=e^{i \delta(\mathbf{k})} \hat{\alpha}_{\lambda}(\mathbf{k})$,

where $\hat{\alpha}_{\lambda}=\hat{\alpha}_{\lambda}^{*}$ are real valued functions. Then we can derive the expectation values of the operators which are diagonal in the basis (81) by using the rule (90) as

$$
\begin{aligned}
\left\langle\left(\mathscr{P}^{i}\right)^{n}\right\rangle & =\frac{1}{\left\langle\alpha\left|\Lambda_{\Delta}\right| \alpha\right\rangle} \int_{\Delta} \mathrm{d}^{3} k\left(k^{i}\right)^{n} \sum_{\lambda} \hat{\alpha}_{\lambda}(\mathbf{k})^{2}, \\
\left\langle\mathscr{W}^{n}\right\rangle & =\frac{1}{\left\langle\alpha\left|\Lambda_{\Delta}\right| \alpha\right\rangle} \int_{\Delta} \mathrm{d}^{3} k \sum_{\lambda} \lambda^{n} \hat{\alpha}_{\lambda}(\mathbf{k})^{2} .
\end{aligned}
$$

For the energy operator, which is not diagonal in this basis, we may apply the same formula but using, in addition, the identity (82) and the Green theorem, which helps us to write

$$
\begin{aligned}
\langle\mathscr{H}\rangle & =\frac{1}{\left\langle\alpha\left|\Lambda_{\Delta}\right| \alpha\right\rangle}\left\{\frac{i \omega_{\mathrm{H}}}{2} \int_{\Delta} \mathrm{d}^{3} k k^{i} \sum_{\lambda} \alpha_{\lambda}^{*}(\mathbf{k}) \stackrel{\leftrightarrow}{\partial} k_{i} \alpha_{\lambda}(\mathbf{k})\right\} \\
& =-\frac{1}{\left\langle\alpha\left|\Lambda_{\Delta}\right| \alpha\right\rangle}\left\{\omega_{\mathrm{H}} \int_{\Delta} \mathrm{d}^{3} k\left[k^{i} \partial_{k^{i}} \delta(\mathbf{k})\right] \sum_{\lambda} \hat{\alpha}_{\lambda}(\mathbf{k})^{2}\right\},
\end{aligned}
$$

since $\hat{\alpha}_{\lambda}^{*} \stackrel{\leftrightarrow}{\partial} \hat{\alpha}_{\lambda}=0$ as these are real valued functions. Note that the operator $k^{i} \partial_{k^{i}}$ in momentum space is in fact a radial operator such that this does not affect the polarization vectors which depend only on the unit vector of the momentum direction, $\mathbf{n}_{k}$. Finally, by using again Eq. (90) we may write

$\left\langle\alpha\left|\mathscr{H}^{2}\right| \alpha\right\rangle=\delta_{i j}\left(\hat{H} A[\alpha]_{i}, \hat{H} A[\alpha]_{j}\right)$,

which helps us to obtain the useful formula

$$
\begin{aligned}
\left\langle\mathscr{H}^{2}\right\rangle= & \frac{\omega_{\mathrm{H}}^{2}}{\left\langle\alpha\left|\Lambda_{\Delta}\right| \alpha\right\rangle}\left\{\int_{\Delta} \mathrm{d}^{3} k\left[k^{i} \partial_{k^{i}} \delta(\mathbf{k})\right]^{2} \sum_{\lambda} \hat{\alpha}_{\lambda}(\mathbf{k})^{2}\right. \\
& \left.+\int_{\Delta} \mathrm{d}^{3} k \sum_{\lambda}\left[\left(k^{i} \partial_{k^{i}}+\frac{3}{2}\right) \hat{\alpha}_{\lambda}(\mathbf{k})\right]^{2}\right\}
\end{aligned}
$$

which we need in the next application.

\section{Quantum redshift}

Let us come back now to the problem of two translated observers, $O^{\prime}$ and $O$, preparing and measuring a photon state $|\alpha\rangle$. We assume that these observers use the same global ideal apparatus represented by the operator algebra $\mathbb{A}^{\prime} \cup \mathbb{A}$ formed by two sub-algebras including the field operators and the conserved ones for which we use the self-explanatory notations

$O^{\prime}: \quad \mathscr{A}^{\prime}\left(x_{\mathrm{c}}^{\prime}\right), \ldots \mathscr{H}^{\prime}, \mathscr{P}^{\prime i}, \mathscr{Q}^{\prime i}, \mathscr{L}_{i}^{\prime} \ldots \in \mathbb{A}^{\prime}$,

$O: \quad \mathscr{A}\left(x_{\mathrm{c}}\right), \ldots \mathscr{H}, \mathscr{P}^{i}, \mathscr{Q}^{i}, \mathscr{L}_{i} \ldots \in \mathbb{A}$.

The state $|\alpha\rangle$ is prepared at the initial time (30) when the observers are translated each other with the position vector d. The translation generators $\mathscr{P}^{i}=\mathscr{P}^{i}$, which are the same in both the above sub-algebras, define the translation operator

$\mathscr{T}(\mathbf{d})=\exp \left(i d^{i} \mathscr{P}^{i}\right)$

which transforms these sub-algebras, $\mathscr{T}(\mathbf{d}): \mathbb{A}^{\prime} \rightarrow \mathbb{A}$, such that any operator $\mathscr{X}^{\prime} \in \mathbb{A}^{\prime}$ is transformed into the operator $\mathscr{X}=T(\mathbf{d}) \mathscr{X}^{\prime} T(\mathbf{d})^{\dagger} \in \mathbb{A}$. Particularly, the energy operator is translated as

$\mathscr{H}=\mathscr{T}(\mathbf{d}) \mathscr{H}^{\prime} \mathscr{T}(\mathbf{d})^{\dagger}=\mathscr{H}^{\prime}+\omega_{\mathrm{H}} d^{i} \mathscr{P}^{i}$, 
according to the commutation rule (84). In what follows, we simplify the geometry be choosing an orthogonal frame $\left\{\mathbf{e}_{1}, \mathbf{e}_{2}, \mathbf{e}_{3}\right\}$ such that $\mathbf{d}=d \mathbf{e}_{3}$.

\subsection{Expectation values}

The observer $O^{\prime}$ prepares the state $|\alpha\rangle$ in his proper frame $\left\{t^{\prime}, \mathbf{x}^{\prime}\right\}_{O^{\prime}}$ where the principal parameters are the expectation values of energy, $E^{\prime}$, momentum components, $P^{\prime i}$, and polarization, $W^{\prime}$. These quantities can be calculated by using the simple rule (90) taking into account that the packet is defined by the functions (94) whose phase must be fixed according to the condition (43). The simplest choice is

$\delta(\mathbf{k})=-\frac{k}{\omega_{\mathrm{H}}}$,

since then the expectation value of the energy reads

$$
\begin{aligned}
E^{\prime} \equiv\left\langle\alpha\left|\mathscr{H}^{\prime}\right| \alpha\right\rangle & =\delta_{i j}\left(A[\alpha]_{i}, \hat{H} A[\alpha]_{j}\right) \\
& =\int \mathrm{d}^{3} k k \sum_{\lambda} \hat{\alpha}_{\lambda}(\mathbf{k})^{2},
\end{aligned}
$$

as it results from Eq. (97) for $\Delta=\mathbb{R}_{k}^{3}$. The other expectation values can be derived simpler by using Eq. (90) as

$$
\begin{aligned}
P^{\prime i} \equiv\left\langle\alpha\left|\mathscr{P}^{i}\right| \alpha\right\rangle & =\delta_{i j}\left(A[\alpha]_{i}, \hat{P}^{i} A[\alpha]_{j}\right) \\
& =\int \mathrm{d}^{3} k k^{i} \sum_{\lambda} \hat{\alpha}_{\lambda}(\mathbf{k})^{2}, \\
W^{\prime} \equiv\left\langle\alpha\left|\mathscr{W}^{i}\right| \alpha\right\rangle & =\delta_{i j}\left(A[\alpha]_{i}, \hat{W} A[\alpha]_{j}\right) \\
& =\int \mathrm{d}^{3} k \sum_{\lambda} \lambda \hat{\alpha}_{\lambda}(\mathbf{k})^{2},
\end{aligned}
$$

since these do not depend on the phase (104). We observe that in our framework where we set the phase (104) all these expectation values have the same forms as in Minkowski spacetime.

However, these quantities are not accessible to the observer $O$ which focuses on the observables, $\mathscr{H}^{\prime}, \mathscr{H}, \mathscr{P}^{i}$ and $\mathscr{W}$ selecting only the photons coming from the source $O^{\prime}$, whose momenta are parallel with $\mathbf{e}_{3}$. This means that the domain of momenta measured by $O$ is

$\Delta=\left\{\mathbf{k} \mid-\frac{\Delta k}{2} \leq k^{1} \leq \frac{\Delta k}{2}, \quad-\frac{\Delta k}{2} \leq k^{2} \leq \frac{\Delta k}{2}, k^{3}<0\right\}$, where $\Delta k$ is a small quantity. Then we may evaluate the integrals over $\Delta$ as

$$
\begin{aligned}
\int_{\Delta} \mathrm{d}^{3} k F(\mathbf{k}) & =\int_{-\frac{\Delta k}{2}}^{\frac{\Delta k}{2}} \mathrm{~d} k^{1} \int_{-\frac{\Delta k}{2}}^{\frac{\Delta k}{2}} \mathrm{~d} k^{2} \int_{-\infty}^{0} \mathrm{~d} k^{3} F\left(k^{1}, k^{2}, k^{3}\right) \\
& \simeq(\Delta k)^{2} \int_{0}^{\infty} \mathrm{d} k F(0,0,-k),
\end{aligned}
$$

according to the mean value theorem.

Now we come back to our intuitive notations of Sect. 3 of the expectation values of the initial, $E_{i} \equiv\left\langle\mathscr{H}^{\prime}\right\rangle$, and final, $E_{f} \equiv\langle\mathscr{H}\rangle$, energies related to the conserved momentum of components $P^{i} \equiv\left\langle\mathscr{P}^{i}\right\rangle$ that can be observed by $O$. These expectation values have to be calculated according to Eq. (92) with the state $|\alpha\rangle$ defined by the functions (94) with the phase (104) and the projection operator $\Lambda_{\Delta}$ corresponding to the domain (108). First we find that

$\left\langle\alpha\left|\Lambda_{\Delta}\right| \alpha\right\rangle=\int_{\Delta} \mathrm{d}^{3} k \sum_{\lambda} \hat{\alpha}_{\lambda}(\mathbf{k})^{2}=(\Delta k)^{2} \kappa$

where

$\kappa=\int_{0}^{\infty} \mathrm{d} k \sum_{\lambda} \hat{\alpha}_{\lambda}(0,0,-k)^{2}$.

Furthermore, we calculate the expectation values defined by Eq. (95) for $n=1$,

$$
\begin{aligned}
P^{3} \equiv\left\langle\mathscr{P}^{3}\right\rangle & =-\frac{1}{\kappa} \int_{0}^{\infty} \mathrm{d} k k \sum_{\lambda} \hat{\alpha}_{\lambda}(0,0,-k)^{2}, \\
\left\langle\mathscr{P}^{1}\right\rangle & =\left\langle\mathscr{P}^{2}\right\rangle=0
\end{aligned}
$$

which do not depend on the phase (104). For the energy operators the situation is different since their expectation values depend on this phase as in Eq. (97) which allows us to write

$E_{i} \equiv\left\langle\mathscr{H}^{\prime}\right\rangle=\frac{1}{\kappa} \int_{0}^{\infty} \mathrm{d} k k \sum_{\lambda} \hat{\alpha}_{\lambda}(0,0,-k)^{2}=-P^{3}$,

deriving the expectation value of Eq. (103) as

$E_{f} \equiv\langle\mathscr{H}\rangle=\left(1-\omega_{\mathrm{H}} d\right) E_{i}$,

recovering thus the Lemaitre form of Hubble's law (33). Note that this result can be derived in a different manner observing that

$$
\langle\alpha|\mathscr{H}| \alpha\rangle=\left\langle\alpha\left|T(\mathbf{d}) \mathscr{H}^{\prime} T(\mathbf{d})^{\dagger}\right| \alpha\right\rangle=\left\langle\tilde{\alpha}\left|\mathscr{H}^{\prime}\right| \tilde{\alpha}\right\rangle,
$$


where now the translated state $|\tilde{\alpha}\rangle$ is given by the functions (94) in which we must substitute

$\delta(\mathbf{k}) \rightarrow \tilde{\delta}(\mathbf{k})=-\frac{k}{\omega_{\mathrm{H}}}-\mathbf{k} \cdot \mathbf{d}$.

With this new phase Eq. (97) gives just the result (115). We must specify that now the initial energy $E_{i}$ observed by $O$ is different from $E^{\prime}$ measured by $O^{\prime}$ in contrast with the classical approach where these two quantities coincide (as in the table of Sec. 3).

In our experiment we select only the momenta oriented along $\mathbf{e}_{3}$ such that the polarizations vectors

$\mathbf{e}\left(\mathbf{e}_{3}, \pm 1\right)=\frac{1}{\sqrt{2}}\left(\mathbf{e}_{1} \mp i \mathbf{e}_{2}\right)$,

are in the plane $\left\{\mathbf{e}_{1}, \mathbf{e}_{2}\right\}$. The expectation value of the PauliLubanski operator,

$W \equiv\langle\mathscr{W}\rangle=\frac{1}{\kappa} \int_{0}^{\infty} \mathrm{d} k\left[\hat{\alpha}_{1}(0,0,-k)^{2}-\hat{\alpha}_{-1}(0,0,-k)^{2}\right]$,

suggests us to introduce the polarization angle $0 \leq \theta(k) \leq \frac{\pi}{2}$ such that

$\hat{\alpha}_{1}(0,0, k)=\cos \theta(k) \alpha(k), \quad \hat{\alpha}_{-1}(0,0, k)=\sin \theta(k) \alpha(k)$

where the new function $\alpha(k)$ satisfies $\int_{0}^{\infty} \mathrm{d} k \alpha(k)^{2}=\kappa$. In the particular case when $\theta$ is a constant independent on $k$ we have $W=\cos 2 \theta$.

\subsection{Dispersions and uncertainty}

The next step is to study the dispersions of the observables measured by $O$ applying the well-known rule

$\operatorname{disp} \mathscr{X}=(\Delta \mathscr{X})^{2}=\left\langle\mathscr{X}^{2}\right\rangle-\langle\mathscr{X}\rangle^{2}$.

We observe first that the operators $\mathscr{H}^{\prime}$ and $\mathscr{H}$ commute alike with $\mathscr{P}^{i}$ and $\mathscr{W}$ as in Eqs. (84) and (85) but do not commute with each other since

$$
\left[\mathscr{H}^{\prime}, \mathscr{H}\right]=i \omega_{\mathrm{H}}^{2} d^{i} \mathscr{P}^{i}=i \omega_{\mathrm{H}}^{2} \mathrm{~d} \mathscr{P}^{3} .
$$

Therefore, from the above equation and Eq. (84) we obtain the uncertainty relations

$$
\begin{aligned}
\operatorname{disp} \mathscr{H}^{\prime} \operatorname{disp} \mathscr{P}^{i} & \geq \frac{1}{4} \omega_{\mathrm{H}}^{2}\left|\left\langle\mathscr{P}^{i}\right\rangle\right|^{2}, \\
\operatorname{disp} \mathscr{H} \operatorname{disp} \mathscr{P}^{i} & \geq \frac{1}{4} \omega_{\mathrm{H}}^{2}\left|\left\langle\mathscr{P}^{i}\right\rangle\right|^{2}, \\
\operatorname{disp} \mathscr{H}^{\prime} \operatorname{disp} \mathscr{H} & \geq \frac{1}{4} \omega_{\mathrm{H}}^{4} d^{2}\left|\left\langle\mathscr{P}^{3}\right\rangle\right|^{2} .
\end{aligned}
$$

For $i=1,2$ Eq. (113) allows us to set $\operatorname{disp} \mathscr{P}^{1}=$ $\operatorname{disp} \mathscr{P}^{2}=0$ without violating the uncertainty relations but along the third axis the relation (123) is non-trivial since $P \equiv\left|\left\langle\mathscr{P}^{3}\right\rangle\right| \neq 0$. On the other hand, from Eq. (99) with the phase (104) we obtain

$$
\left\langle\mathscr{H}^{\prime 2}\right\rangle=\left\langle\left(\mathscr{P}^{3}\right)^{2}\right\rangle+\omega_{\mathrm{H}}^{2} \chi .
$$

The last term of the above equation represents the quantum correction which is proportional with the dimensionless quantity

$$
\chi=\frac{1}{\kappa} \int_{0}^{\infty} d k \sum_{\lambda}\left[\left(k \partial_{k}+\frac{3}{2}\right) \hat{\alpha}_{\lambda}(0,0,-k)\right]^{2},
$$

resulting from the last term of Eq. (99). This is generated by the de Sitter gravity and depends exclusively on the form of the functions $\hat{\alpha}_{\lambda}$. For deriving the dispersion of the operator $\mathscr{H}$ we calculate first the expectation value (99) with the new phase (117) obtaining the identity

$\left\langle\mathscr{H}^{2}\right\rangle=\left(1-\omega_{\mathrm{H}} d\right)^{2}\left\langle\left(\mathscr{P}^{3}\right)^{2}\right\rangle+\omega_{\mathrm{H}}^{2} \chi$.

Finally, from Eqs. (126) and (128) combined with Eqs. (114) and (115) we find

$$
\begin{aligned}
\operatorname{disp} E_{i} & \equiv \operatorname{disp} \mathscr{H}^{\prime}=\operatorname{disp} P+\omega_{\mathrm{H}}^{2} \chi, \\
\operatorname{disp} E_{f} & \equiv \operatorname{disp} \mathscr{H}^{2}=\left(1-\omega_{\mathrm{H}} d\right)^{2} \operatorname{disp} P+\omega_{\mathrm{H}}^{2} \chi,
\end{aligned}
$$

where we denote $\operatorname{disp} P \equiv \operatorname{disp} \mathscr{P}^{3}$.

Now we come back to the uncertainty relations (123) and (124) by using Eqs. (129) and (130) for deriving the inequalities

$$
\begin{aligned}
\operatorname{disp} E_{i, f}\left(\operatorname{disp} E_{i, f}-\omega_{\mathrm{H}}^{2} \chi\right) & \geq \frac{1}{4} \omega_{\mathrm{H}}^{2} E_{i, f}^{2}, \\
\operatorname{disp} P\left(\operatorname{disp} P+\omega_{\mathrm{H}}^{2} \chi\right) & \geq \frac{1}{4} \omega_{\mathrm{H}}^{2} P^{2},
\end{aligned}
$$


from which we deduce

$$
\begin{aligned}
\operatorname{disp} E_{i, f} & \geq \frac{\omega_{\mathrm{H}}}{2}\left(\sqrt{E_{i, f}^{2}+\omega_{\mathrm{H}}^{2} \chi^{2}}+\omega_{\mathrm{H}} \chi\right) \\
& =\frac{\omega_{\mathrm{H}}}{2}\left(E_{i, f}+\omega_{\mathrm{H}} \chi+\frac{1}{2 E_{i, f}} \omega_{\mathrm{H}}^{2} \chi^{2}\right)+\mathscr{O}\left(\omega_{\mathrm{H}}^{4} \chi^{4}\right), \\
\operatorname{disp} P & \geq \frac{\omega_{\mathrm{H}}}{2}\left(\sqrt{P^{2}+\omega_{\mathrm{H}}^{2} \chi^{2}}-\omega_{\mathrm{H}} \chi\right) \\
& =\frac{\omega_{\mathrm{H}}}{2}\left(P-\omega_{\mathrm{H}} \chi+\frac{1}{2 P} \omega_{\mathrm{H}}^{2} \chi^{2}\right)+\mathscr{O}\left(\omega_{\mathrm{H}}^{4} \chi^{4}\right),
\end{aligned}
$$

relating thus the dispersions to the corresponding expectation values. More interesting is Eq. (125) as depending explicitly on the distance $d$ between $O$ and $O^{\prime}$. This can be rewritten in our new notation as

$\operatorname{disp} E_{i} \operatorname{disp} E_{f} \geq \frac{1}{4} \omega_{\mathrm{H}}^{4} d^{2} P^{2}$,

and can be seen as the starting point for deriving new inequalities depending on $d$ by using Eqs. (129) and (130). Our preliminary calculations indicate that these are more complicated requiring a special analytical a numerical study which will be performed elsewhere.

Finally, let us analyze the dispersion of the Pauli-Lubanski operator in the simple case when the polarization angle $\theta$ is independent on $k$. We have seen that then the expectation value has the form $W \equiv\langle\mathscr{W}\rangle=\cos 2 \theta$. Moreover, from Eq. (96) we obtain

$\left\langle\mathscr{W}^{2}\right\rangle=1 \rightarrow \operatorname{disp} \mathscr{W}=\sin ^{2} 2 \theta$,

while from Eq. (85) we derive the uncertainty relation

$\operatorname{disp} \mathscr{H} \operatorname{disp} \mathscr{W} \geq \frac{1}{4} \omega_{\mathrm{H}}^{2}|\langle\mathscr{W}\rangle|^{2}$

giving the restriction

$\tan 2 \theta \geq \frac{1}{2} \frac{\omega_{\mathrm{H}}}{\Delta E_{f}}$,

preventing one from measuring total polarizations, i.e. $\theta=0$ for $\lambda=1$ or $\theta=\frac{\pi}{2}$ for $\lambda=-1$.

\section{Concluding remarks}

We presented the complete classical and quantum theory of the Maxwell field minimally coupled to the gravity of the de Sitter expanding universe focusing on the principal effect due to the space expansion, namely the redshift for which we derived the quantum corrections and the principal uncertainty relations.
In the actual expanding universe the quantum corrections and the limits of the uncertainty relations are extremely small, since the actual value of $\omega_{\mathrm{H}}$ (or $\hbar \omega_{\mathrm{H}}$ in SI units) is of the order $10^{-33} \mathrm{eV}$ such that it is less probably identified in astronomic observations. Moreover, the limitation predicted by the inequality (138) in an ideal universe is too small to be separated from other polarization effects produced by the cosmic dust and plasma.

However, these results are interesting as coming from the first complete and coherent classical and quantum theory of the Maxwell field coupled to the gravity of an expanding universe. The methods developed here can be applied to any spatially flat FLRW expanding universe including the actual models of early universe.

On the other hand, the method of regularization of the momentum dependent phase plays the same role as the rest frame vacuum of the massive particles ensuring the correct flat limit of the Maxwell field. Thus we obtain a coherent quantum theory on the de Sitter expanding universe in which we may apply the perturbation methods of the traditional quantum theory in Minkowski spacetime. For this reason we hope that our approach will open the door to a large field of applications not only in astrophysics and cosmology, but even in particle physics.

Data Availability Statement This manuscript has no associated data or the data will not be deposited. [Authors' comment: This article is theoretical without using or producing numerical data.]

Open Access This article is licensed under a Creative Commons Attribution 4.0 International License, which permits use, sharing, adaptation, distribution and reproduction in any medium or format, as long as you give appropriate credit to the original author(s) and the source, provide a link to the Creative Commons licence, and indicate if changes were made. The images or other third party material in this article are included in the article's Creative Commons licence, unless indicated otherwise in a credit line to the material. If material is not included in the article's Creative Commons licence and your intended use is not permitted by statutory regulation or exceeds the permitted use, you will need to obtain permission directly from the copyright holder. To view a copy of this licence, visit http://creativecomm ons.org/licenses/by/4.0/.

Funded by $\mathrm{SCOAP}^{3}$.

\section{References}

1. E.R. Harrison, Cosmology: The Science of the Universe (Cambridge University Press, New York, 1981)

2. G.E. Lemaître, Ann. Soc. Sci. de Bruxelles 47A, 49 (1927)

3. G.E. Lemaître, MNRAS 91, 483 (1931)

4. E. Hubble, Proc. Natl. Acad. Sci. 15, 168 (1929)

5. E. Harrison, Astrophys. J. 403, 28 (1993)

6. I.I. Cotăescu, Eur. Phys. J. C 77, 485 (2017)

7. I.I. Cotăescu, Eur. Phys. J. C 78, 95 (2018)

8. I.I. Cotăescu, Mod. Phys. Lett. A 36, 2150022 (2021)

9. I.I. Cotăescu, Eur. Phys. J. C 81, 32 (2021)

10. I.I. Cotăescu, arXiv:2101.02019

11. I.I. Cotăescu, C. Crucean, Prog. Theor. Phys. 124, 1 (2010) 
12. I.I. Cotăescu, C. Crucean, Phys. Rev. D 87, 044016 (2013)

13. I.I. Cotăescu, GRG 43, 1639 (2011)

14. P. Painleve, C. R. Acad. Sci. (Paris) 173, 677 (1921)

15. I.I. Cotăescu, Mod. Phys. Lett. A 22, 2965 (2007)

16. I.I. Cotăescu, C. Crucean, A. Pop, Int. J. Mod. Phys. A 23, 2563 (2008)

17. I.I. Cotăescu, Int. J. Mod. Phys. A 35, 2030019 (2020)

18. N.D. Birrel, P.C.W. Davies, Quantum Fields in Curved Space (Cambridge University Press, Cambridge, 1982)

19. I.I. Cotăescu, Mod. Phys. Lett. A 32, 1750223 (2017)

20. I.I. Cotăescu, arXiv:2102.03211, accepted for publication in Chinese. Phys
21. I.I. Cotăescu, Eur. Phys. J. C 79, 696 (2019)

22. I.I. Cotăescu, Eur. Phys. J. C 80, 621 (2020)

23. I.I. Cotăescu, Eur. Phys. J. C 80, 535 (2020)

24. I.I. Cotăescu, Chin. Phys. C 45, 1 (2021)

25. O. Nachtmann, Commun. Math. Phys. 6, 1 (1967)

26. S. Weinberg, The Quantum Theory of Fields (Cambridge University Press, Cambridge, 1995)

27. I.I. Cotăescu, Phys. Rev. D 65, 084008 (2002)

28. A. Messiah, Quantum Mechanics (Dover, New York, 1999) 